\title{
Early- and Late-Time Behavior of Attractors in Heavy-Ion Collisions
}

\author{
Aleksi Kurkela, ${ }^{1,2, *}$ Wilke van der Schee, ${ }^{1, \dagger}$ Urs Achim Wiedemann $\oplus^{1, *}$ and Bin Wu ${ }^{1, \S}$ \\ ${ }^{1}$ Theoretical Physics Department, CERN, CH-1211 Genève 23, Switzerland \\ ${ }^{2}$ Faculty of Science and Technology, University of Stavanger, 4036 Stavanger, Norway
}

(Received 22 July 2019; revised manuscript received 20 December 2019; accepted 4 February 2020; published 10 March 2020)

\begin{abstract}
Whether, how, and to what extent solutions of Bjorken-expanding systems become insensitive to aspects of their initial conditions is of importance for heavy-ion collisions. Here we study $1+1 \mathrm{D}$ and phenomenologically relevant boost-invariant $3+1 \mathrm{D}$ systems in which initial conditions approach a universal attractor. In Israel-Stewart theory (IS) and kinetic theory where the universal attractor extends to arbitrarily early times, we show that all initial conditions approach the attractor at early times by a power law while their approach is exponential at late times. In these theories, the physical mechanisms of hydrodynamization operational at late times do not drive the approach to the attractor at early times, and the early-time attractor is reached prior to hydrodynamization. In marked contrast, the attractor in strongly coupled systems is realized concurrent with hydrodynamization. This qualitative difference may offer a basis for discriminating weakly and strongly coupled scenarios of heavy-ion collisions.
\end{abstract}

DOI: $10.1103 /$ PhysRevLett.124.102301

In a dynamical system, an attractor is the subset of phase space to which arbitrary initial conditions within the basin of attraction relax at sufficiently late times. In general, the attractor is characterized by the competition between the expansion rate that drives the system towards local anisotropy, and the isotropizing interaction rate [1]. Attractors are easily found empirically by evolving a set of different initial conditions (see Fig. 1 for an example). Recently, such attractors have received attention in the context of ultrarelativistic heavy-ion collisions. Their form is of interest for understanding the onset of fluid-dynamic behavior [2-21] and the origin of the nonthermal fixed-point behavior in far-from-equilibrium dynamics [1,19,22-25]. For the phenomenology of heavy-ion collisions, these studies are needed to clarify to what extent different observables inform us either about the details of the initial conditions or about the material properties of the system.

Whether an attractor exists at arbitrarily early times depends on the dynamics that drives the initial conditions to the attractor. Here we point out that some models undergoing Bjorken expansion do exhibit attractor behavior at arbitrarily early times while others do not. The existence of the early-time attractor is a consequence of the longitudinal expansion at early times which would render heavy-ion phenomenology insensitive to the unknown details of the longitudinal structure of the initial state.

Published by the American Physical Society under the terms of the Creative Commons Attribution 4.0 International license. Further distribution of this work must maintain attribution to the author(s) and the published article's title, journal citation, and DOI. Funded by SCOAP .
Israel-Stewart theory.-In Bjorken-expanding IsraelStewart (IS) theory [26] with transverse translational symmetry, an attractor exists for the ratio of longitudinal pressure $p_{L}$ over energy density $\varepsilon$,

$$
\begin{gathered}
\partial_{\tau} \varepsilon+\frac{1}{\tau}\left[\varepsilon+p_{L}\right]=0, \\
\partial_{\tau} \phi+\frac{4 \phi}{3} \frac{\phi}{\tau}=-\frac{1}{\tau_{R}}\left[\phi-\frac{4}{3} \frac{\eta}{\tau}\right] .
\end{gathered}
$$

Here, $\tau$ is the proper time and $\phi \equiv \frac{1}{3} \varepsilon-p_{L}$. The time governing the relaxation to fluid-dynamic constitutive equations is $\tau_{R}=(5 / a)(\eta /(\varepsilon+P))$, where $a$ is a free parameter, conventionally fixed to $a=1$. This is the timescale on which linearized nonhydrodynamic excitations decay.

We work for a conformal equation of state $\varepsilon=3 P$ and constant specific viscosity $\eta / s \propto \eta / \varepsilon^{3 / 4}$. The equation of motion for the ratio $x \equiv p_{L} / \varepsilon$ (written for convenience in the rescaled time $\left.t=\tau / \tau_{R}\right)$ reads

$$
\left(\frac{3}{4}-\frac{x}{4}\right) \frac{d x}{d t}=\frac{45 x^{2}-30 x+5+[15 t(1-3 x)-16 a]}{45 t} .
$$

The limit $a \rightarrow 0$ at finite $\tau_{R}$ is equivalent to an ideal IS theory with $\eta=0$. In this simplest case, the attractor is the equilibrium $x_{A}=1 / 3$, and how the attractor is approached is given by rewriting Eq. (3) in terms of the deviation $\delta=x-x_{A}$, 

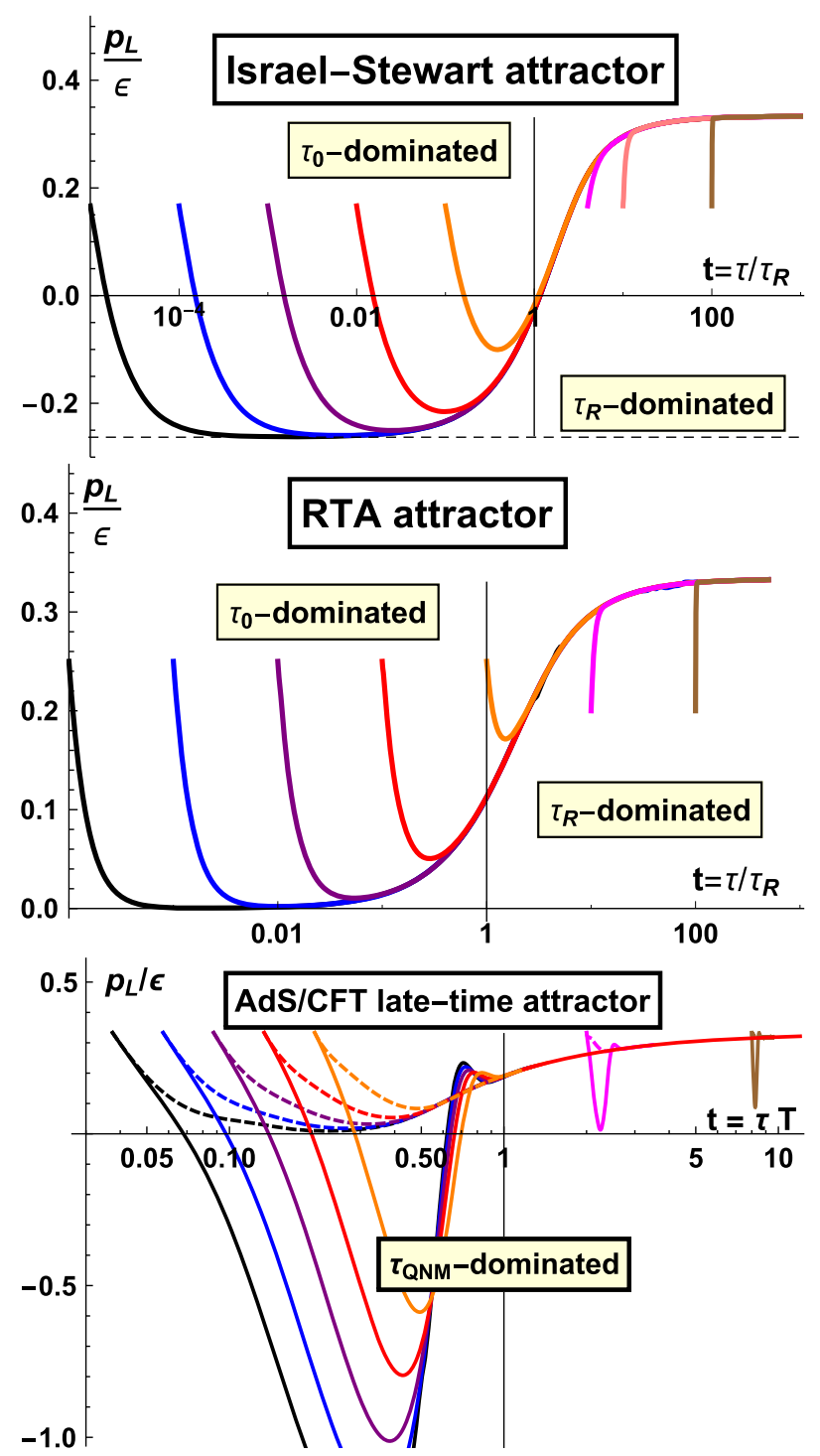

FIG. 1. Approach to the attractor in different theories. Different lines correspond to out-of-attractor initializations at different times $t_{0}$. Upper and middle panel: For $t<1$, the slope of the approach remains constant on a log-linear scale, indicating that the time dependence is set by the initialization time $t_{0}$ and thus governed by the expansion rate. In contrast, for $t>1$, the approach to the attractor appears on a log-linear scale steeper and steeper with increasing $t$, indicating its dependence on the interaction rate $\tau_{R}$ which does not depend on $t_{0}$. Lower panel: Qualitatively different behavior is seen for $\mathcal{N}=4$ SYM theory, where information characteristic of specific initial condition is lost only at times $t \geq 1$, irrespective of how early the system is initialized.

$$
\left(\frac{3}{4}-\frac{1+3 \delta}{12}\right) \frac{d \delta}{d t}=\frac{\delta^{2}}{t}-\delta
$$

Depending on whether $t>\delta$ or $t<\delta$, the approach to the attractor is governed by the expansion rate $\left(t^{-1}\right)$ or by the interaction rate (independent of $t$ ), respectively. At all times, sufficiently small deviations from the attractor decay exponentially $\delta \sim e^{-3 t / 2}$, which is characteristic for linearized nonhydrodynamic perturbations around thermal equilibrium [6]. The factor $3 / 2$ arises from the nontrivial time evolution of the background.

For finite $a$, Eq. (3) corresponds to the first-order IS theory, which has two solutions that remain regular for $t_{0} \rightarrow 0$ with limits $\lim _{t_{0} \rightarrow 0} x_{ \pm}\left(t_{0}\right)=(1 / 15)(5 \pm 4 \sqrt{5 a})$, respectively. [We note that within IS theory, $a$ is a free parameter. The choice $a=1$ amounts to equating $\tau_{R}$ to the second order hydrodynamic coefficient $\tau_{\pi}$ of RTA kinetic theory. The choice $a=5 / 16$ would instead ensure that the early-time attractor of IS theory coincides with that of kinetic theory, $\left.x_{-}\left(t_{0}\right)=0\right]$. The solution $x_{-}(t)$ is the attractor $x_{A}(t)$, while $x_{+}(t)$ limits the basin of attraction from above. While we do not have an analytic solution $x_{-}(t)$, the attractor can be expanded at late and early times, see Supplemental Material [27] for details. The main finding, see Fig. 2, is that the early-time expansion is a convergent series that can be analytically continued to arbitrarily late times using standard techniques, while the late-time expansion is a nonconvergent, asymptotic Borelresummable series. We next discuss the transient dynamics that evolves generic initial conditions from time $t_{0}$ towards the attractor. At late initializations, $t_{0} \gg 1$, this is the wellknown exponential decay of linearized nonhydrodynamic modes governed by Eq. (4) that, as discussed above, is determined by the interaction rate, see Fig. 1. Exponential decays with this timescale have been revealed in Borel resummations of the late time expansion [6]. In marked contrast, at early times, Eq. (3) becomes

$$
\left(\frac{3}{4}-\frac{x_{A}(t=0)+\delta}{4}\right) \frac{d \delta}{d t}=\frac{\delta}{t}\left[\frac{15 \delta-8 \sqrt{5 a}}{15}\right],
$$

and the attractor is approached by a power law

$$
\delta \sim t^{-8 \sqrt{5 a} /(\sqrt{5 a}+10)} .
$$

We emphasize that the timescale of this decay becomes increasingly rapid and ultimately instantaneous with decreasing $t_{0}$ (see Fig. 1). This is the hallmark of a decay governed by the expansion rate. It is qualitatively different from what one expects from the decay of nonhydrodynamic modes, and it forces the decay to the attractor prior to hydrodynamization.

Higher-order fluid-dynamic models like rBRSSS [31] amount to replacing in Eq. (2) the relaxation to the first order constitutive relation (the term $\frac{4}{3}(\eta / s)$ ) by relaxation to the second order one. In general, all additional terms thus introduced are $\propto(1 / \tau)$. As a consequence, the value of the early-time attractor changes, but the early-time power-law approach of arbitrary initial conditions towards the attractor is unaffected. 

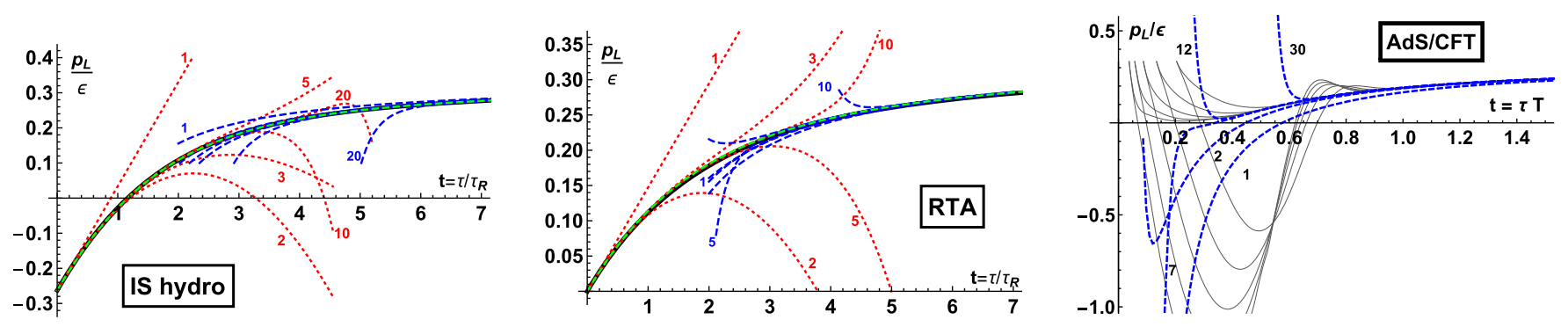

FIG. 2. Early-time (red-dashed) and late-time (blue-dashed) expansions of the attractor (black). Orders of the expansions are given by numbers in the plots. The Padé approximant (green-dashed) extends the early-time expansion to any finite $t$ in systems where the early-time attractor exists. For $\mathcal{N}=4 \mathrm{SYM}$, gray curves are the specific solutions shown in Fig. 1.

Kinetic theory.-Features similar to the above can also be seen in Bjorken-expanding massless kinetic theory in the relaxation time approximation (RTA)

$$
\partial_{\tau} f+\vec{v}_{\perp} \cdot \partial_{\vec{x}_{\perp}} f-\frac{p_{z}}{\tau} \partial_{p_{z}} f=-\frac{\left(-v_{\mu} u^{\mu}\right)}{\tau_{R}}\left[f-f_{\mathrm{eq}}\right]
$$

Here, the distribution function $f\left(\tau, \vec{x}_{\perp} ; \vec{p}_{\perp}, p_{z}\right)$ relaxes to equilibrium $f_{\text {eq. }}$. It depends on $p^{\mu}=\left(p, \vec{p}_{\perp}, p_{z}\right), p=$ $\sqrt{\vec{p}_{\perp}^{2}+p_{z}^{2}}$, and on the proper time $\tau ; u_{\mu}$ denotes the rest frame of the energy density and $\vec{v}_{\perp}=\vec{p}_{\perp} / p, v_{z}=p_{z} / p$ are transverse and longitudinal velocities, respectively. We work with a conformal relaxation time $\tau_{R}^{-1}=\gamma \varepsilon^{1 / 4}$.

For systems with transverse translational symmetry, this Boltzmann equation can be reduced to a tower of moment equations $[8,18]$ describing the time evolution of various integral moments of the distribution function $p_{l} \equiv$ $\int_{-1}^{1}\left(d v_{z} / 2\right) \int\left(4 \pi d p p^{3} /(2 \pi)^{3}\right) f v_{z}^{2 l}$. Energy density and longitudinal pressure correspond to the first two moments, $\varepsilon=p_{0}, p_{L}=p_{1}$. The first two equations in the hierarchy result in

$$
\left(\frac{3}{4}-\frac{x}{4}\right) \frac{d x}{d t}=\frac{3 x^{2}-6 x+3 y+t(1-3 x)}{3 t},
$$

where $y \equiv p_{2} / \varepsilon$; see Supplemental Material [27] for further details. The attractor is found among the regular solutions. Solutions that remain regular for $t_{0} \rightarrow 0$ satisfy $\lim _{t_{0} \rightarrow 0} x_{ \pm}\left(t_{0}\right)=1 \pm \sqrt{1-y(0)}$. All physical values $x \leq 1$ lie within the basin of attraction since $x_{+}>1$. Since $0<y<x$, the attractor at early times is $x_{A}(0)=0$, and therefore also all higher moments $p_{l}(0)$ vanish. At late times, it follows trivially from Eq. (8) that the attractor approaches equilibrium, $\lim _{t \rightarrow \infty} x_{A}(t)=\frac{1}{3}$.

The late-time fluid-dynamic expansion of this transport theory has been computed to high orders [20]. Similar to IS theory, it is a nonconvergent, asymptotic Borelresummable series. Also, similarly, the early-time expansion has a finite radius of convergence and can be extended to arbitrary late times by standard techniques (see Supplemental Material [27]).
The transient dynamics according to which generic initial conditions approach this attractor shares the main qualitative features of the IS theory discussed above, see Fig. 1. At late initializations, $t_{0} \gg 1$, Eqs. (8) and (4) govern identical exponential decays of linearized nonhydrodynamic modes. For early times, $t_{0} \ll 1$, the decay of $\delta=x-x_{A}$ to the attractor depends on $y$, and through $y$ on the initial conditions of all higher moments. Because $6 x>3 y$ for any system, an approximate solution of the approach to the attractor can be obtained for a generic initial condition by neglecting $y$ in Eq. (8) which leads to the power-law decay $\delta \sim t^{-8 / 3}$. Similar reasoning suggests that $y$ would approach its attractor $\sim t^{-16 / 3}$ thus justifying the above approximation; in fact, the same reasoning gives for all higher orders $p_{l}(t) / \varepsilon(t) \propto x(t)^{l}$. These power laws are easily seen in numerical solutions of Eq. (7) (see Supplemental Material [27]).

Strongly coupled $\mathcal{N}=4$ SYM.-The third class of qualitatively different models of collectivity invoked in heavy-ion physics is given by strongly coupled quantum field theories with known gravity duals. Here, we contrast and compare the early-time dynamics in strongly coupled $\mathcal{N}=4$ super Yang-Mills (SYM) theory with the attractor behavior observed above. The time evolution of $p_{L} / \epsilon$ can be solved for boost invariant initial conditions using standard methods of holography, i.e., by solving the five-dimensional Einstein equations with the ansatz for the line-element

$d s^{2}=-2 \rho^{-2} d \rho d \tau-A d \tau^{2}+S^{2} e^{B} d \mathbf{x}_{\perp}^{2}+S^{2} e^{-2 B} d \xi^{2}$,

with $\xi$ the space-time rapidity and $\rho$ the internal fifth dimension. The initial conditions are specified by the $\rho$ dependent function $B\left(\tau_{0}, \rho\right)=B_{\text {AdS }}\left(\tau_{0}, \rho\right)+B_{0}(\rho)$, with $B_{\text {AdS }}\left(\tau_{0}, \rho\right)=-\frac{2}{3} \log \left(\tau_{0}+\rho\right)$ the vacuum anti-de Sitter (AdS) solution; $B(\tau, \rho), A(\tau, \rho)$, and $S(\tau, \rho)$ then follow from the Einstein equations [32]. Unlike in the models above changing the initial Cauchy data $B_{0}(\rho)$ amounts to a choice not only for $\left(p_{L}\left(t_{0}\right) / \varepsilon\left(t_{0}\right)\right)$, but also for all its derivatives at $t_{0}[3,33]$. We therefore study two different families of initial conditions, $B_{0}^{(\mathrm{UV})}(\rho)=e^{-40 \rho T} 32 \rho^{5} T^{5}$ and 
$B_{0}^{(\mathrm{IR})}(\rho)=32 \rho^{5} T^{5}$, where $T$ is the effective temperature determined from the energy density. While their functional form is somewhat arbitrary, they are chosen such that their initial anisotropy $\left(p_{L}\left(t_{0}\right) / \varepsilon\left(t_{0}\right)\right)$ and its first derivative are equal. They differ qualitatively in that their support is either localized close to the boundary (UV) or spread out in the fifth dimension (IR). As an aside, we recall that also kinetic theory can be initialized with different classes of initial conditions by varying the values of higher moments at $t_{0}$, but all initial conditions approach the same attractor on a timescale $t_{0}$, see Supplemental Material [27]. In IS theory, there are no further degrees of freedom beyond $\left(p_{L}\left(t_{0}\right) / \varepsilon\left(t_{0}\right)\right)$ that can be specified.

Figure 1 shows solutions in which both initial conditions are evolved from a set of different initialization times $t_{0}$. In marked contrast to IS theory and kinetic theory, different initial conditions do not reach a unique curve on timescale $t_{0}$. Rather, information about the initial condition is lost only at times $t \sim 1$, and only on that timescale solutions converge to a common attractor. By closer inspection of these results (data not shown) we observe that both for early initializations $t_{0}<1$ and for late initializations $t_{0}>1$, differences between solutions show the oscillatory behavior characteristic for the decay of quasinormal modes (QNM) [16] that are exponentially damped with timescale $\tau_{\mathrm{QNM}}$. These solutions reach a unique attractor only at late times $t>1$. In this sense, only the late time attractor is universal in $\mathcal{N}=4$ SYM; this is consistent with Fig. 2 of Ref. [9].

It is curious to note that curves initialized with $B_{0}^{(\mathrm{UV})}(\rho)$ approach the high-order hydrodynamic late-time expansion [5] significantly earlier than curves initialized with $B_{0}^{(\mathrm{IR})}(\rho)$, see Fig. 2. The latter initial condition is expected to give rise to a larger connected two-point function $\left\langle T^{\alpha \beta} T^{\mu \nu}\right\rangle$ than the former [34]. We believe that this observation, together with vanishing $n$-point functions in the above kinetic theory and in IS theory, should motivate further research into the relation of higher connected $n$ point functions and attractor behavior. This question could be asked not only in $\mathcal{N}=4$ SYM, but also in BBGKY extensions of the Boltzmann equation.

Attractors in boost-invariant 3+1D kinetic theory.Would an early-time attractor, if it exists, leave observable imprints? If so, this could provide a tool for disentangling qualitatively different microscopic candidate theories of weakly or strongly coupled quark gluon plasma. With this motivation, we now ask which aspects of the attractor behavior are accessible in collisions with a finite transverse extent and realistic transverse gradients. We focus on the kinetic theory (7) as it possesses an early-time attractor. We have solved Eq. (7) for realistic initial transverse profiles [35]. Because the early-time approach to the attractor is governed by the longitudinal expansion rate, breaking the translational symmetry in the transverse directions can change the $1+1 \mathrm{D}$ picture only to the extent to which transverse gradients are not negligible compared to the longitudinal one. Therefore, at sufficiently early initialization, independent of the transverse geometry and for all transverse positions $r$, arbitrary initial conditions in $3+1 \mathrm{D}$ evolve towards the $1+1 \mathrm{D}$ attractor. In contrast, the latetime evolution of the attractor does depend on the transverse profile of energy and transverse momentum.

These features are realized in boost-invariant $3+1 \mathrm{D}$ solutions of Eq. (7), initialized with a Gaussian transverse energy profile with central energy density $\varepsilon_{0}$ and r.m.s. radius $R$, see Fig. 3. For early initialization time $\tau_{0}$, keeping $\varepsilon_{0} \tau_{0}$ fixed, Eq. (7) can be rescaled such that the evolution depends on only one dimensionless combination of model parameters, the opacity $\hat{\gamma}=\gamma R^{3 / 4}\left(\varepsilon_{0} \tau_{0}\right)^{1 / 4}=$ $\left(\gamma^{3} \varepsilon_{0}^{3 / 4} R^{3} t_{0}\right)^{1 / 4}$, see Refs. [35,36]. The opacity of a system increases with coupling strength $(\gamma)$, transverse system size $(R)$, and initial central energy density $\left(\varepsilon_{0}\right)$; physical collision systems were estimated to correspond to a range of opacities, $\hat{\gamma} \lesssim 2$ for proton-nucleus collision, $2 \lesssim \hat{\gamma} \lesssim 4$ for semiperipheral $\mathrm{PbPb}$ collisions and somewhat higher values in central $\mathrm{PbPb}$ collisions [35].

The physical time in Fig. 3 is rescaled by a position- and time-dependent relaxation time $\tau_{R}^{-1}(\tau, r)=\gamma \varepsilon(\tau, r)^{1 / 4}$. Therefore, for a system in which energy density decreases faster than $\propto \tau^{-4}$ due to transverse expansion, the relation between physical and rescaled time is not monotonic; this is the reason $t$ decreases for sufficiently late $\tau$ in the finite- $\hat{\gamma}$ curves of Fig. 3. Moreover, because of this rescaling, the deviation of the $r=0$ attractor from the $1+1 \mathrm{D}$ one, and the deviation of the attractor at finite $r$ from the one at $r=0$ arise solely from the radial expansion. For fixed $\hat{\gamma}$, the $r$ dependence is remarkably mild. Low orders in the earlytime expansion are seen to be sufficient to describe systems characterized by values of $\hat{\gamma}$ that are within experimental

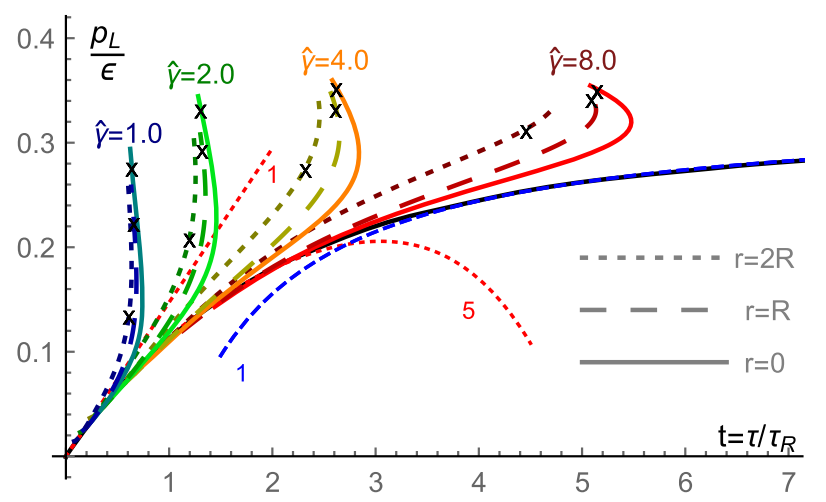

FIG. 3. RTA attractor of Bjorken-expanding $3+1 \mathrm{D}$ kinetic theory for collision systems of different opacity $\hat{\gamma}$ and three different transverse positions $r=0, R$, and $2 R$. The $1+1 \mathrm{D}$ attractor (black line) corresponds to the limit of infinite opacity; the thin lines correspond to early- and late-time approximations, as in Fig. 2. The black crosses denote the point on the attractor where the physical time reaches $\tau=2 R$ after which the system has decoupled [35]. 
reach. What Fig. 3 makes abundantly clear is that what remains universal across collision geometries is not the late-time attractor but the early-time attractor. That is, what remains universal is what follows from early-time dynamics and not what follows from hydrodynamization.

In summary, we have studied the early-time behavior of qualitatively different models of collectivity applied to heavy-ion collisions. In some cases $(\mathcal{N}=4$ SYM $)$, the attractor exists only at late times, and hydrodynamization and the loss of information about specific initial conditions are concurrent. The approach to the late-time attractor is then governed by the exponential decay of linearized nonhydrodynamic modes $[2,16]$. In other cases (IS and kinetic theory) a unique attractor extends to arbitrarily early times and thus specific information about the initial condition is lost well before hydrodynamization. We find that in this latter case, a qualitatively different power-law approach to the attractor is operational far from equilibrium. It is expansion driven rather than interaction driven. It is also noteworthy that in this respect, IS theory at any value of $\eta / s$ resembles RTA - the prototype of a weakly coupled system-rather than the prototypical strongly coupled system of $\mathcal{N}=4$ SYM.

One of the main challenges in heavy ion phenomenology is to elucidate the inner workings of the quark gluon plasma, and in particular, to discriminate between weakly and strongly coupled plasma models in which a quasiparticle picture exists or does not exist, respectively. The qualitative difference stated here between the early-time dynamics of strongly coupled $(\mathcal{N}=4 \mathrm{SYM})$, compared to both kinetic theory and IS theory, deserves attention since it may help to make this distinction.

*a.k@cern.ch

†wilke.van.der.schee@cern.ch

*urs.wiedemann@cern.ch

§b.wu@cern.ch

[1] A. Kurkela and G. D. Moore, J. High Energy Phys. 11 (2011) 120.

[2] R. A. Janik and R. B. Peschanski, Phys. Rev. D 74, 046007 (2006).

[3] G. Beuf, M. P. Heller, R. A. Janik, and R. Peschanski, J. High Energy Phys. 10 (2009) 043.

[4] M. P. Heller, R. A. Janik, and P. Witaszczyk, Phys. Rev. Lett. 108, 201602 (2012).

[5] M. P. Heller, R. A. Janik, and P. Witaszczyk, Phys. Rev. Lett. 110, 211602 (2013).

[6] M. P. Heller and M. Spalinski, Phys. Rev. Lett. 115, 072501 (2015).
[7] L. Keegan, A. Kurkela, P. Romatschke, W. van der Schee, and Y. Zhu, J. High Energy Phys. 04 (2016) 031.

[8] G. S. Denicol and J. Noronha, arXiv:1608.07869.

[9] P. Romatschke, Phys. Rev. Lett. 120, 012301 (2018).

[10] M. Spaliński, Phys. Lett. B 776, 468 (2018).

[11] M. Strickland, J. Noronha, and G. S. Denicol, Phys. Rev. D 97, 036020 (2018).

[12] A. Behtash, C. N. Cruz-Camacho, and M. Martinez, Phys. Rev. D 97, 044041 (2018).

[13] J. P. Blaizot and L. Yan, Phys. Lett. B 780, 283 (2018).

[14] M. P. Heller and V. Svensson, Phys. Rev. D 98, 054016 (2018).

[15] G. S. Denicol and J. Noronha, Phys. Rev. D 99, 116004 (2019).

[16] M. Spaliński, Phys. Lett. B 784, 21 (2018).

[17] A. Behtash, S. Kamata, M. Martinez, and H. Shi, Phys. Rev. D 99, 116012 (2019).

[18] M. Strickland and U. Tantary, J. High Energy Phys. 10 (2019) 069.

[19] A. Kurkela and Y. Zhu, Phys. Rev. Lett. 115, 182301 (2015).

[20] M. P. Heller, A. Kurkela, M. Spaliński, and V. Svensson, Phys. Rev. D 97, 091503(R) (2018).

[21] P. Romatschke, J. High Energy Phys. 12 (2017) 079.

[22] J. Berges, K. Boguslavski, S. Schlichting, and R. Venugopalan, Phys. Rev. D 89, 114007 (2014).

[23] J. Berges, K. Boguslavski, S. Schlichting, and R. Venugopalan, Phys. Rev. Lett. 114, 061601 (2015).

[24] A. Mazeliauskas and J. Berges, Phys. Rev. Lett. 122, 122301 (2019).

[25] K. Boguslavski, A. Kurkela, T. Lappi, and J. Peuron, Phys. Rev. D 100, 094022 (2019).

[26] W. Israel and J. M. Stewart, Ann. Phys. (N.Y.) 118, 341 (1979).

[27] See Supplemental Material at http://link.aps.org/ supplemental/10.1103/PhysRevLett.124.102301 for details of the IS and RTA series expansions shown in Fig. 2, which includes Refs. [28-30].

[28] M. Strickland, Acta Phys. Pol. B 50, 1243 (2019).

[29] N. Borghini and C. Gombeaud, Eur. Phys. J. C 71, 1612 (2011).

[30] P. Romatschke, Eur. Phys. J. C 78, 636 (2018).

[31] R. Baier, P. Romatschke, D. T. Son, A. O. Starinets, and M. A. Stephanov, J. High Energy Phys. 04 (2008) 100.

[32] P. M. Chesler and L. G. Yaffe, Phys. Rev. D 82, 026006 (2010).

[33] M. P. Heller, R. A. Janik, and P. Witaszczyk, Phys. Rev. D 85, 126002 (2012).

[34] C. Ecker, D. Grumiller, and S. A. Stricker, J. High Energy Phys. 07 (2015) 146.

[35] A. Kurkela, U. A. Wiedemann, and B. Wu, Eur. Phys. J. C 79, 965 (2019).

[36] A. Kurkela, U. A. Wiedemann, and B. Wu, Phys. Lett. B 783, 274 (2018). 